\title{
CARACTERIZACIÓN GENÉTICA DE SEIS MICROSATÉLITES EN EQUINOS COLOMBIANOS Equus caballus (Equidae) Y SU USO EN PRUEBAS DE FILIACIÓN
}

\author{
GENETIC CHARACTERIZATION OF SIX \\ MICROSATELLITES IN COLOMBIAN HORSES \\ Equus caballus (Equidae) AND ITS USE FOR \\ PARENTAGE TESTING
}

Alexandra Gómez Tarazona ${ }^{1}$

Fernando Ariza Botero ${ }^{2}$

Ligia Jiménez Robayo ${ }^{3}$

\begin{abstract}
RESUMEN
Los equinos colombianos son una especie de gran importancia social, cultural y comercial, pero el desconocimiento de su potencial genético ha limitado los programas de selección y de mejoramiento. Para dar inicio a este proceso, se evaluó la variabilidad genética de 264 caballos pertenecientes a las razas equinas caballo criollo colombiano, paso fino colombiano y trocha pura colombiana. La caracterización alélica, se hizo con seis marcadores moleculares tipo microsatélite (ASB17, AHT4, AHT5, HTG4, HMS3 y HMS6) para un posterior estudio poblacional. El análisis genético de los seis microsatélites indica alto polimorfismo alélico para el locus ASB17 con 18 alelos, siendo el menos variable es el HTG4, con siete alelos; el número promedio de
\end{abstract}

\footnotetext{
${ }^{1}$ Médico Veterinario M.Sc. U.N. Instituto de Genética. U.N. e.mail: nagomezt@unal.edu.co Dirección para correspondencia: Entrada Calle 53 con Cra. 37, Edificio 426, Of. 216.

${ }^{2}$ Médico Veterinario, M.Sc., PhD. Fac. de Medicina Vetrinaria y de Zootecnia, U.N.

${ }^{3}$ Médico Veterinario, M.Sc. Fac. de Medicina Vetrinaria y de Zootecnia, U.N.
}

alelos fue de 9,83. Las frecuencias alélicas muestran que las poblaciones están representadas por pocos alelos. Para determinar la variabilidad genética en la población, se calculó la diversidad génica $\mathrm{H}_{\mathrm{e}}$ 0,773 y $\mathrm{H}_{\mathrm{o}}$ 0,765 indicando una alta variabilidad. Por otra parte, se encontró una alta diversidad genética en los caballos de Cundinamarca, con un coeficiente de endogamia $\mathrm{F}_{\text {IS }}$ 0,0011. Para los casos de filiación, los loci AHT4 y HMS3 demostraron ser los más informativos por sus altos valores en la probabilidad de exclusión; sin embargo, el valor de probabilidad acumulada de 0,9543 con los seis marcadores relativamente bajo. Se recomienda cambiar los marcadores AHT4 y HMS6 por otros más informativos y aumentar el número de marcadores en el caso de su uso en pruebas de paternidad.

Palabras clave: equinos colombianos, diversidad genética, microsatélites, frecuencias alélicas.

\section{SUMMARY}

In terms of commercial and cultural implications Colombian equines are very valuable; however their genetic potential is unknown. As a result, programs regarding 
selection and improvement have been limited. In order to contribute to the genetic potential, the variability of 264 horses Criollo Colombiano, Paso Fino Colombiano y Trocha Pura Colombiana breeds was evaluated. The allelic characterization was made with six molecular markers, micro satellite sort (ASB17, AHT4, AHT5, HTG4, HMS3 y HMS6) for further population studies. The analysis of the six microsatellites indicated high allelic polymorphism for the ASB17 with 18 alleles, the less variable was the HTG4 with seven alleles. The allele's standard was 9.83. Besides, the allelic frequency showed that the populations are represented by few alleles. The gene diversity $\mathrm{H}_{\mathrm{e}}$ 0.7773 and $\mathrm{H}_{\mathrm{o}} 0.765$ was calculated to determine the genetic variability of the population. It indicated a large variability and high gene diversity in equines from the department of Cundinamarca with an endogamy coefficient of $\mathrm{F}_{\text {IS }}$ 0.0011. In the paternity test, the AHT4 and HMS3 demonstrated that they are the most informative ones; which is due to their high exclusion probability. Nevertheless, the accumulated probability validity of 0.9543 with the six markers was low. It is recommended to change the AHT4 and HMS6 markers for more informative ones and also to increase the number of markers.

Key words: Colombian horses, genetic diversity, microsatellites, allelic frequencies.

\section{INTRODUCCIÓN}

Los caballos colombianos están conformados por varias razas: criollo colombiano, paso fino colombiano, trocha pura colombiana y trote-galope reunido colombiano. Información sobre estas razas se encuentra en el banco de datos de la Organización para la Agricultura y la Alimentación (FAO, 2004). Estas razas son de origen español y se respalda con informaciones históricas de los viajes de Colón y otros conquistadores (Rodero et al. 1992) y con estudios de ADN mitocondrial que han determinado las relaciones filogenéticas del caballo español con algunas razas suramericanas (Mirol et al. 2002). Una vez fueron introducidos los caballos españoles, se dispersaron y se adaptaron a los diversos ambientes de Suramérica. Las consecuencias de esto son posibles modificaciones genotípicas y fenotípicas para el caballo asentado en el Nuevo Mundo. Las características fenotípicas del caballo colombiano y su buen desempeño deportivo hacen que sea una de las especies domésticas de gran reconocimiento e importancia económica, generando altos ingresos por su comercialización.
Las caracterizaciones sirven para establecer la situación actual de la variabilidad genética en una población, ya que las presiones demográficas y económicas pueden generar procesos que llevan al detrimento de las razas y algunas especies (FAO, 1997); así mismo, los procedimientos reproductivos en animales de granja, como los cruzamiento dirigidos, el apareamiento consanguíneo y la selección artificial pueden interferir en la variabilidad genética (Ron et al. 1996). En la especie equina, la caracterización de diferentes razas se ha realizado a nivel morfológico (Herrera E Navarrete, 1978) y molecular permitiendo describir las razas genéticamente (Cañon et al. 2000; Rodas, 2000). Igualmente, los marcadores han permitido establecer las diferencias entre ellas (Achmann et al. 2004), conocer la variabilidad genética (Kavar et al. 1999, Tozaki et al. 2003, Romero, 2000) y proponer procesos de manejo y selección de los recursos genéticos (Zabek et al. 2005; Ron et al. 1996), así como para el mantenimiento y conservación del patrimonio genético (Fernández et al. 2004, Ruiz- García et al. 2003).

Los marcadores moleculares microsatélites (STR) son repeticiones cortas que funcionan como loci co-dominantes, se heredan de forma mendeliana, son altamente polimorficos y están dispersos abundantemente en el genoma nuclear de los organismos eucariotes (Hancock, 1999; Weber \& May, 1989). Los microsatélites han sido usados en las ciencias forenses en pruebas de filiación de caballos silvestres (Bowling, 1997), bovinos (Glowatzki-Mullis et al. 1995) y caninos (Cho E Cho, 2003) y en el control del linaje al mantener la rutina de la genotipificación de las poblaciones animales (Chikhi et al. 2004).

En otras especies, los microsatélites han permitido la elaboración de mapas de ligamiento (Womack, 2005), la evaluación del parentesco (Tozaki et al. 2001, Luikart et al. 1999) y la evaluación de susceptibilidad a enfermedades (Carrington et al. 1999).

Los caracterizaciones de los caballos colombianos en su gran mayoría han sido descripciones morfológicas (Virgüez, 2004, Estrada, 1995, Vivas, 1994 y Herrera E Navarrete, 1978) y siendo los estudios genéticos con marcadores moleculares en equinos colombianos escasos (Romero, 2000, Rodas, 2000). Además, estos no han determinado la estructura de la población equina colombiana. En este contexto, se realizó la genotipificación del ADN con seis marcadores microsatélites para 
la identificación individual y verificación de filiación de caballos colombianos. Así mismo, se analizó la diversidad genética y se calculó el contenido de información polimórfica (PIC) y la probabilidad de exclusión (PE).

\section{MATERIALES Y MÉTODOS}

Muestra de la población: Se colectaron muestras de sangre periférica de la vena yugular de 132 caballos no emparentados de la raza criollo colombiana, en Cundinamarca. Y otras 132 muestras corresponden a los registros de tipificación de equinos en Cundinamarca de las razas paso fino colombiano y trocha pura, registrados en Federación Colombiana de Asociaciones Equinas FEDEQUINAS.

Extracción de ADN y amplificación PCR: La genotipificación fue hecha con seis marcadores microsatélites recomendados para las pruebas de filiación por la Sociedad Internacional de Genética Animal (ISAG). Estos marcadores fueron AHT4 y AHT5 (Binns et al. 1995), ASB17, HMS3 y HMS6 y HTG4 (Ellegren et al.1992). El ADN fue extraído por la técnica de fenol-cloroformo. Los seis microsatélites fueron amplificados solos en seis reacciones de PCR. Las reacciones de PCR fueron realizadas en un volumen final de $15 \mu \mathrm{L}$ conteniendo $2 \mu \mathrm{L}$ de DNA, con: 3,0 $\mu \mathrm{L}$ de ADN, $200 \mu \mathrm{M}$ de solución de dNTPs (Promega, USA), $10 \mathrm{mM}$ de buffer $(100 \mathrm{mM}$ Tris- $\mathrm{HCl}, 500 \mathrm{mM} \mathrm{KCl}, 1 \%$ Triton), 0,8 $\mu \mathrm{M}$ de solución de cada primer, el $\mathrm{MgCl}_{2}$ varió entre $1,5 \mathrm{Mm}$ y 2,0Mm y $0.5 \cup$ de Taq polimerasa (CORPOGEN®, Colombia) e iniciadores $20 \mathrm{ng} / \mu \mathrm{L}$. La amplificación fue llevada a cabo en un termociclador (GeneAmp PCR System PTC-100J, PE Applied Biosystems) usando las siguientes condiciones: una denaturación inicial a $95^{\circ} \mathrm{C}$ por $5 \mathrm{~min}$, seguido por 30 ciclos de $95^{\circ} \mathrm{C}$ por 30 s, $64,62,60$ o $59^{\circ} \mathrm{C}$ por $45 \mathrm{~s}$, y $72^{\circ} \mathrm{C}$ por $30 \mathrm{~s}$, la extensión final $72^{\circ} \mathrm{C}$ por $10 \mathrm{~min}$ y estabilización $30^{\circ} \mathrm{C}$ por un minuto. Los microsatélites amplificados fueron separados en gel de poliacrilamida denaturante al $6 \%$, seguido por la tinción de plata. La asignación de los alelos, se llevó a cabo manualmente, con controles positivos de equinos genotipificados previamente por la UC (Davis) y el patrón de peso molecular 10pb a 200pb con 10pb DNA ladder (Invitrogen USA). La nomenclatura de los genotipos, se basó en símbolos del alfabeto español, recomendada para equinos por el Horse Standing Committe of the Internacional Society for Animals Genetics at Tours, France, 1996.
Análisis Estadístico: Las frecuencias alélica fueron calculadas en los diferentes loci y para las dos agrupaciones usando el programa FSTAT (Goudet, 2001). El número total de alelos observados, heterocigocidad observada y diversidad genética, se estimaron para las poblaciones agrupadas usando el programa GENETIX (Belkhir et al. 2000). La desviación del equilibrio Hardy-Weinberg fueron estimadas para los seis loci y por agrupación mediante el programa GENEPOP version 1.2 (Raymond \& Rousset 1995). La estructura genética fue analizada con los estadísticos $F$ de Wright, según el método de Weir E Cockerham (1984) usando FSTAT (Goudet, 2001). Y se llevó a cabo, adicionalmente, un análisis multivariante con el método de análisis de correspondencias múltiples (ACM), el cual se ejecutó con el programa Multivariate Statistical Package (MVSP) 3.1 (http://www.kovcomp.com). Las distancias genéticas se calcularon con la distancia de cuerda de Cavalli-Sforza E Edwards (1967), opción incluida dentro del programa Genetix v.4.03 (Belkhir et al. 2000). Los estimadores de paternidad calculados fueron: la probabilidad de exclusión (PE), la probabilidad de exclusión combinada (PEC) y el contenido de información polimórfica (PIC), mediante el programa CERVUS (Marshall et al. 1998).

\section{RESULTADOS Y DISCUSIÓN}

En los caballos criollos de Cundinamarca, en seis loci, se detectaron 54 alelos, mientras que en la agrupación correspondiente a FEDEQUINAS, se observaron 59 alelos. La diferencia se presentó en los loci ASB17 con 14 y 18 alelos, respectivamente y AHT5 con el alelo $S$ en la agrupación de Fedequinas. El locus de mayor polimorfismo fue ASB17 con 18 alelos, cuatro de éstos de baja frecuencia ( $D, S, T$ y V) y, el menos polimorfico HTG4, con 7 alelos (Tabla 1).

Agrupando los 264 individuos en una sola población, se estimó que el locus con mayor heterocigocidad observada $\left(\mathrm{H}_{\mathrm{o}}\right)$ y esperada $\left(\mathrm{H}_{\mathrm{e}}\right)$ fue ASB17, el de menor $\mathrm{H}_{\mathrm{e}}$ y $\mathrm{H}_{\mathrm{o}}$ fue HMS6 (Tabla 2). El locus HMS3 con ocho alelos mostró una diversidad genética alta 0,811. Las frecuencias alélicas de los seis marcadores indican que algunos alelos son los más frecuentes en la población (Tabla 2).

La comparación de la distribución alélica y genotípica entre los caballos criollos colombianos y los agrupados 
Tabla 1. Frecuencias alélicas de seis microsatélites de la población de caballos colombianos.

\begin{tabular}{|c|c|c|c|c|c|c|}
\hline Alelos & ASB17 & AHT5 & HMS6 & HMS3 & HTG4 & AHT4 \\
\hline D & 0,004 & & & & & \\
\hline \multicolumn{7}{|l|}{$E$} \\
\hline $\mathrm{F}$ & 0,039 & & & & & \\
\hline G & 0,009 & & & & & \\
\hline $\mathrm{H}$ & 0,011 & & 0,049 & & & 0,184 \\
\hline I & 0,014 & 0,007 & & 0,065 & & 0,012 \\
\hline $\mathrm{J}$ & 0,050 & 0,109 & & & & 0,082 \\
\hline $\mathrm{K}$ & 0,006 & 0,175 & 0,093 & & 0,093 & 0,103 \\
\hline$L$ & 0,072 & 0,031 & 0,131 & & 0,170 & 0,110 \\
\hline M & 0,254 & 0,224 & 0,098 & 0,175 & 0,463 & 0,037 \\
\hline $\mathrm{N}$ & 0,080 & 0,315 & 0,007 & 0,298 & 0,125 & 0,045 \\
\hline 0 & 0,049 & 0,077 & 0,077 & 0,170 & 0,116 & 0,388 \\
\hline$P$ & 0,044 & 0,009 & 0,529 & 0,135 & 0,026 & 0,039 \\
\hline$Q$ & 0,073 & 0,054 & 0,016 & 0,127 & 0,007 & \\
\hline $\mathrm{R}$ & 0,233 & & & 0,026 & & \\
\hline$S$ & 0,003 & & & 0,002 & & \\
\hline $\mathrm{T}$ & 0,004 & & & & & \\
\hline U & 0,054 & & & & & \\
\hline V & 0,001 & & & & & \\
\hline $\mathrm{Na}$ & 18 & 9 & 8 & 8 & 7 & 9 \\
\hline $\mathrm{He}$ & 0,852 & 0,798 & 0,676 & 0,811 & 0,718 & 0,780 \\
\hline Ho & 0,799 & 0,797 & 0,697 & 0,776 & 0,745 & 0,775 \\
\hline
\end{tabular}

por FEDEQUINAS no mostró diferencias significativas $(\mathrm{P}<0,05)$. La evaluación de la estructura de las poblaciones con los estadísticos $F$ de Wright (Tabla 3) revelaron poca diferenciación genética $\left(F_{S T}=0,0210\right)$, con un intervalo de confianza entre - 0,00200 y 0,06276 estimado por bootstrapping, con una confianza del 95\%; así mismo pusieron de manifiesto un bajo coeficiente de endogamia $F_{I S}$ 0,011. De otra forma, las distancias de Cavalli-Sforza (Cavalli-Sforza \& Edwards, 1967) para la pareja Cundinamarca-Fedequinas fue de 0,004 y para la distancia de Nei (1987), fue de 0,098.
El análisis de correspondecias multiples (ACM) de las frecuencias alélicas obtenidas de los seis microsatélites en los caballos de Cundinamarca, con las calculadas por Rodas (2000), con 2668 equinos del occidente colombiano y con las obtenidas por Romero (2000) con 400 animales de diferentes zonas de Colombia, determinó que el $71 \%$ de la inercia total se ubica en el primer eje indicando un agrupamiento de las frecuencias alélicas por similitud en los seis loci en los tres estudios en distintas zonas de Colombia. De igual manera, los valores propios de los dos ejes son bajos $(0,108$ y 0,017$)$, todo lo anterior establece que no hay diferencias entre las poblaciones de caballos evaluadas. 
Tabla 2. Frecuencia alélica de seis microsatélites de FEDEQUINAS y de los seis criaderos agrupados de Cundinamarca.

\begin{tabular}{|c|l|l|l|l|l|l|l|l|l|l|l|l|}
\hline \multirow{2}{*}{ Alelos } & \multicolumn{3}{|c|}{ FEDEQUINAS } & \multicolumn{7}{c|}{ CRIADEROS } \\
\cline { 2 - 7 } & AHT5 & HMS6 & HMS3 & HTG4 & AHT4 & ASB17 & AHT5 & HMS6 & HMS3 & HTG4 & AHT4 \\
\hline D & 0,002 & & & & & & & & & & & \\
\hline E & & & & & & & & & & & & \\
\hline F & 0,045 & & & & & & 0,013 & & & & & \\
\hline G & 0,009 & & & & & & 0,013 & & & & & \\
\hline H & 0,010 & & 0,048 & & & 0,183 & 0,009 & & 0,053 & & & 0,189 \\
\hline I & 0,006 & 0,006 & & 0,063 & & 0,010 & 0,018 & 0,009 & & 0,079 & & 0,022 \\
\hline J & 0,058 & 0,110 & & & & 0,081 & 0,053 & 0,105 & & & & 0,088 \\
\hline K & 0,005 & 0,168 & 0,091 & & 0,094 & 0,105 & 0,009 & 0,206 & 0,105 & & 0,088 & 0,092 \\
\hline L & 0,028 & & 0,128 & & 0,167 & 0,109 & 0,009 & & 0,145 & & 0,189 & 0,114 \\
\hline M & 0,290 & 0,007 & 0,098 & 0,183 & 0,470 & 0,036 & 0,289 & 0,145 & 0,101 & 0,136 & 0,430 & 0,044 \\
\hline N & 0,091 & 0,196 & 0,007 & 0,024 & 0,122 & 0,042 & 0,075 & 0,360 & 0,009 & 0,035 & 0,140 & 0,061 \\
\hline O & 0,041 & 0,352 & 0,081 & 0,323 & 0,116 & 0,395 & 0,026 & 0,132 & 0,057 & 0,345 & 0,114 & 0,355 \\
\hline P & 0,047 & 0,092 & 0,536 & 0,136 & 0,027 & 0,039 & 0,088 & 0,004 & 0,496 & 0,132 & 0,022 & 0,035 \\
\hline Q & 0,022 & 0,003 & 0,012 & 0,124 & 0,004 & & 0,026 & 0,039 & 0,035 & 0,145 & 0,018 & \\
\hline R & 0,271 & & & 0,136 & & & 0,325 & & & 0,105 & & \\
\hline S & 0,004 & 0,065 & & 0,009 & & & & & & 0,014 & & \\
\hline T & 0,005 & & & & & & & & & & & \\
\hline U & 0,065 & & & & & & 0,061 & & & & & \\
\hline V & 0,001 & & & & & & & & & & & \\
\hline
\end{tabular}

Tabla 3. Estimación de los estadísticos $\mathrm{F}$ para las poblaciones agrupadas FEDEQUINAS y criaderos.

\begin{tabular}{|l|l|c|}
\hline Estadísticos $\mathbf{F}$ & Valor & Intervalos de Confianza 95\% \\
\hline$F_{I S}$ & $-0,0049$ & $-0,02433-0,01154$ \\
\hline$F_{S T}$ & 0,0233 & $-0,00048-0,06608$ \\
\hline$F_{I T}$ & 0,0184 & $-0,02403-0,07068$ \\
\hline
\end{tabular}

Comparando los estudios efectuados en caballos colombianos sobre los indicadores de variabilidad genética, se encontró que el número promedio de alelos (NMA) fue semejante: en Rodas (2000) 10,25 alelos para 16 loci;
9,14 en Romero (2000) con 14 loci y en el presente estudio fueron 9,80 para seis loci. Adicionalmente, fueron similares los valores de heterocigocidad esperada y observada. 
Se observa la inexistencia del equilibrio Hardy Weinberg en HMS3 $(P<0,000)$ y ASB17 $(p<0,000)$ y se determinó déficit de heterocigotos en HMS3, mientras que los demás loci estuvieron en equilibrio. Las parejas en desequilibrio de ligamiento fueron HMS3 - ASB17 $(0,003)$ y AHT5 - ASB17 $(0,003)$ con una valor de significancia $(\mathrm{p}<0,05)$.
El contenido de información polimórfica (PIC) varió desde 0,799 para ASB17 a 0,679 en HMS6 y el PIC medio fue de 0,743. La probabilidad de exclusión fue mayor para el locus ASB17 y la menor para los loci HTG4 y HMS6, la probabilidad de exclusión acumulada (PEA) de los seis loci fue de 0,954, lo que significa que de 1000 individuos tomados al azar 9540 equinos son excluidos de ser el padre correctamente (Tabla 4).

Tabla 4. Índice F por loci, contenido de información polimórfica y probabilidad de exclusión.

\begin{tabular}{|c|c|c|c|c|c|c|c|}
\hline & ASB17 & HMS6 & HMS3 & HTG4 & AHT4 & AHT5 & Total \\
\hline F & 0,064 & $-0,031$ & 0,044 & $-0,037$ & 0,009 & 0,002 & 0,011 \\
\hline PE & 0,434 & 0,347 & 0,450 & 0,347 & 0,452 & 0,397 & 0,954 \\
\hline PIC & 0,799 & 0,694 & 0,791 & 0,684 & 0,751 & 0,756 & 0,743 \\
\hline
\end{tabular}

F- Índices de fijación. PIC contenido de información polimorfica. PE Probabilidad de exclusión

Los equinos de Cundinamarca representan a la población equina colombiana que esta constituida por las razas caballo criollo colombiano, paso fino colombiano y trocha pura colombiana. La población colombiana de caballos es homogénea por ser genéticamente similar en las diferentes regiones y altamente variable en los seis microsatélites estudiados, con una heterocigocidad observada $\mathrm{H}_{\mathrm{O}} 0,65$, levemente inferior a la diversidad genética $\mathrm{H}_{\mathrm{e}} 0,773$.

El desequilibrio Hardy-Weinberg, se explica por la sustitución de una base en la secuencia flanqueante del marcador HMS3 descrito por (Achmann et al. 2001) y su efecto es de homocigocidad. Esta reducción calculada de heterocigotos es errada e introducida por la falla en la amplificación del marcador HMS3. Esta misma condición logra explicar la asociación estadística detectada el desequilibrio de ligamiento con este marcador.

Las distancias genéticas y los estadísticos F de Wright pusieron de manifiesto que los caballos que se agrupan en FEDEQUINAS (paso fino y el caballo de trocha) y los caballos criollos evaluados de Cundinamarca conforman una misma población. El $F_{S T} 2,32 \%$ ratifica la similaridad por la escasa diferenciación genética entre las agrupa- ciones, mientras que los análisis de varianza a nivel de los individuos, con respecto a cada agrupación y a la población total $\left(F_{I S}\right.$ y $\left.F_{I T}\right)$, son bastante bajos (Tabla 3 ). Indicando que la endogamia en la población es muy baja, con escasa diferenciación genética, sin afectar la variabilidad genética.

Los resultados muestran que la población de caballos colombianos es una sola e indica una variación genética relativamente alta estimada por la diversidad genética y la heterocigocidad observada. Comparando la variabilidad genética calculada de cuatro microsatélites con el Caballo Uruguayo (Kelly et al. 2002), los equinos colombianos tienen una variabilidad levemente superior en el número de alelos, en la heterocigocidad observada y en la diversidad genética. Cotejando la diversidad genética obtenida en los caballos colombianos con la obtenida en las razas españolas fundadoras, como el Lusitano $\mathrm{He}=0,735$ (Luis et al. 2006), y razas gallegas 0,750 (Cañon et al. 2000), los equinos en Colombia mantienen la diversidad genética, la cual, se ha podido dar por diferentes motivos. Estos son: primero porque la dispersión de caballos hacia América es reciente, aproximadamente cinco siglos atrás; segundo, por la multiplicidad de orígenes geográficos de las razas espa- 
ñolas; tercero, por los niveles bajos de selección artificial entre las razas equinas y cuarto, por la influencia de los factores demográficos, como el mantenimiento de los animales en las áreas tradicionalmente dispuestas y poco uso en labores del campo.

Así mismo, la variabilidad genética es similar también a la de otras razas equinas europeas, como el caballo Noruego (Bjornstad et al. 2000). Sin embargo es superior cuando se compara con razas equinas que padecen un manejo reproductivo aislado y tienen barreras geográficas, como en los caballos salvajes Przewalski (Aberle et al. 2004), Lippizzian (Achman et al. 2004) y semi silvestres, como Menorquina, Jaca, Navarra (Glazko, 1998; Solís et al. 2005), los cuales tienen bajo polimorfismo y baja variabilidad genética.

Los microsatélites HTG4 y HMS6 son inadecuados para la pruebas de filiación por su baja probabilidad de exclusión (PE), por lo cual se deben usar un mayor número de microsatélites y más polimórficos para aumentar la PEA, como lo efectuado en otras razas equinas (Tozaki et al. 2003; Kakoi et al. 1998; Meriaux et al. 1998) y otras especies (Giovambattista et al. 2001; StahlbergerSaitbekova et al. 2001; Luikart et al. 1999) y así ser útiles en las pruebas de filiación.

La divergencia entre los genomas de los animales domésticos y el uso microsatélites altamente polimorficos ha permitido la identificación y la asignación de raza en diferentes especies, como los bovinos (MacHugh et al. 1998), los ovinos (Buchanan et al. 1994) y en algunas razas equinas (Bjornstad \& Roed, 2001). Para las razas equinas colombianas la asignación por raza con seis microsatélites no es posible por ser genéticamente homogéneas, sin embargo, aumentar el número de marcadores podría mejorar el poder de discriminación entre las razas colombianas.

\section{CONCLUSIONES}

El uso de los microsatélites recomendados por la FAO para la caracterización genética permitió evaluar y comparar la diversidad genética de los caballos colombianos con otras razas, como las que han influido en la formación de las razas colombianas. Este estudio muestra un análisis genético de seis marcadores en la raza de caballos criollos colombianos con morfología y con características de producción similares. De igual manera, se determinó que las razas equinas colombianas, conformadas por los caballos criollos colombianos, el paso fino colombiano y los caballos trocha colombiana, no se diferencian genéticamente entre ellas, conformando una sola población equina. La variación genética de los caballos colombianos integrados por las tres razas antes mencionadas es alta comparada con otras razas equinas y no hay pérdida de la variabilidad genética.

\section{AGRADECIMIENTOS}

Agradecemos a Beatriz Salgado por el suministro de los datos de FEDEQUINAS. El estudio fue soportado por la Corporación Colombiana de Investigación Agropecuaria (CORPOICA).

\section{BIBLIOGRAFÍA}

ACHMANN, R.; CURIK, I.; DOVC, P.; KAVAR, T.; BODO, I.; HABE, F.; MARTI, E.; SÖLKNER, J.; BREM, G. 2004. Microsatellite diversity, population subdivision and gene flow in the Lipizzan horse. Animal Genetics. 35:285-292.

ACHMANN, R.; HUBER, T.; WALLNER, B.; DOVC, P.; MÜLLER, M.; BREM, G. 2001. Base substitutions in the sequences flanking microsatellite markers HMS3 and ASB2 interfere with parentage testing in the Lipizzan horse. Animal Genetics 32:52.

ABERLE, K.S.; HAMANN, H.; DRÖGEMÜLLER, C.; DISTL, O. 2004. Genetic diversity in German draught horse breeds compared with a group of primitive, riding and wild horses by means of microsatellite DNA markers. Animal Genetics 35:270-277.

BELKHIR, K. 2003. DetSel 1.0: a computer program to detect markers responding to selection. J. Hered. 94(5):429-431.

BELKHIR, K.; BORSA, P.; GOUDET, J.; CHIKHI, L.; BONHOMME, F. 2000. GENETIX 4.02, logiciel sous Windows TM pour la génétique des populations. Laboratoire Génome, Populations, Interactions: CNRSUPR. 9060. Université de Montpellier II, Montpellier, France. 
BINNS, MM.; HOLMES, NG.; HOLLIMAN, A.; SCOTT, AM. 1995. The identification of polymorphic microsatellite loci in the horse and their use in Thoroughbred parentage testing. British Vet. J. 151:9-15.

BJORNSTAND, G.; GUNNBY, E.; ROED, K.H. 2000. Genetic structure of Norweigan horse breeds. J. Animal Breeding and Genetics 117:307-317.

BJORNSTAND, G.; ROED, K.H. 2001. Breed demarcation and potential for breed allocation of horses assessed by microsatellite markers. Animal Genetics. 32:59-65.

BOWLING, AT. 1997 Horse Genetics. Eds. CAB International Oxford UK. p.45-58.

BUCHANAN. F.C.; ADAMS. L.J.; LITTLEJOHN. R.P.; MADDOX. J.F; CRAWFORD. A.M. 1994. Determination of evolutionary relationships among sheep breeds using microsatellites. Genomics 22: 397-403.

CAÑON, J.; CHECA, M.; CARLEOS. C.; VEGA-PLA, J.L.; VALLEJO, M.; DUNNER, S. 2000. The genetic structure of Spanish Celtic horse breeds inferred from microsatellite data. Animal Genetics.31:3948.

CARRINGTON, M.; MARTI, D.; WADE, J.; KLITZ, W.; BARCELLOS, L.; THOMPSON, G.; CHEN, J.; TRUEDSSON, L.; STURFELT, G. 1999. Microsatellite markers in complex disease: Mapping disease-associated regions within the human major histocompatibility complex. En: OXFORD Univ. Press. Microsatellites: Evolution and Applications. p.225 236.

CAVALLI-SFORZA, L.L.; EDWARDS, A.W.F. 1967. Phylogenetic analysis: models and estimation procedures. Am. J. Human Genetics 19:233-257.

CHIKHI, L.; GOOSSENS, B.; TREANOR, A.; BRUFORD, M.W. 2004. Population genetic structure of and inbreeding in an insular cattle breed, the Jersey, and its implications for genetic resource management. Heredity. 92:396-401.
CHO, GJ.; CHO, BW. 2003. Validation of microsatellite markers for routine canine parentage testing in Korea. Korean J. Genet. 25:103-108.

ELLEGREN, H.; ANDERSSON, L.; JOHANSSON, M.; SANDBERG, K. 1992. DNA fingerprint in horses using simple (TG)n probe and its application to population comparisons. Animal Genetics. 23:1-9.

ESTRADA, L.R. 1995. Los caballos criollos colombianos también tienen historia. Revista Fedequinas. 2:56-60.

FAO (Organización para la Agricultura y la Alimentación). 1997. The global strategy for the management of Farm Animal Genetic Resources: A call for action. FAO.Publishing Rome. p.10-13

FAO (Organización para la Agricultura y la Alimentación). 2004. Domestic Animals Diversity, Disponible desde Internet en: http://www.fao.org/dad.is/ (con acceso 10/02/05).

FERNÁNDEZ, J.; TORO, M.; CABALLERO, A. 2004. Managing individuals' contributions to maximize the allelic diversity maintained in small, conserved populations. Conservation Biology. 18(5):1358-1367

GIOVAMBATTISTA, G.; RIPOLI, M.V.; LIRON, J.P.; VILLEGAS-CASTAGNASSO, E.E.; PERAL-GARCIA, P; LOJO, M.M. 2001. DNA typing in a cattle stealing case. J. Forensic Sci. 46(6):1484-1486.

GLAZKO, M. 1998 XXVIth International Conference on Animal Genetics. Auckland, NZ. Section A. p.25.

GLOWATZKI-MULLIS, ML.; GAILLARD, C.; WIGGER, G.; FEIES, R. 1995. Microsatellite-based parentage control in cattle. Anim Genet. 26, 7-12.

GOUDET, J. 2001. Disponible desde Internet en: http:// www.unil.ch/izea/softwares/fstat.html (con acceso 07/03/05).

HANCOCK, J.M. 1999. Microsatellites and other simple sequences: genomic context and mutational mecanisms. En: Goldstein, D.B.; Schlötterer, C. edits. Microsatellites: Evolution and Apllications. Oxford Univ. Press. p.1-23. 
HERRERA, J.; NAVARRETE, A. 1978. Descripción y evaluación de las características raciales del caballo criollo. Tesis de grado. Facultad de Medicina Veterinaria y de Zootecnia. Universidad Nacional de Colombia. Bogotá. p.54-62.

INTERNATIONAL SOCIETY ANIMAL SCIENCES (ISAG). 1996. Mvalues and Chromosomes Assignaments for STRs Reported by VGL. Veterinary Genetic Laboratory, School of Veterinary Medicine, University of California, Davis, CA. Disponible desde Internet en: http://www.vgl.ucdavis.edu/ /vmillon/mvalues. html (con acceso 15/05/05).

KELLY, L.; POSTIGLIONI, D.F.; DEANDRÈS, D.F.; VEGAPLA, GAGLIARDI, R.; BIAGETTI, R.; FRANCO, J. 2002. Genetic haracterization of the Uruguayan creole horses and analysis of relationships among horse breeds. Res. Vet. Sci. 72:69-73.

KAKOI, T.; TOZAKI, S.; HIROTA, K. 1998. Evaluation of a new microsatellite markers for horse parentage testing. XXVI ${ }^{\text {th }}$ International Conference on Animal Genetics. Auckland, NZ. Section A. p.24.

KAVAR, T.; HABE, F.; BREM, G.; DOVC, P. 1999. Mitochondrial $\mathrm{D}$-loop sequence variation among the 16 maternal lines of the Lipizzan horse breed. Animal Genetics. 30:423-430.

LUIS, C.; BASTOS-SILVEIRA, C.; COTHRAN, E.G.; MAR-OOM, M. 2006. Iberian Origins of New World Horse Breeds. J. Hered. 97(2):107-113.

LUIKART, G,; BIJU-DUVAL, M-P.; ERTUGRUL, O.; ZAGDSUREN, Y.; MAUDET, C.; P TABERLET. 1999. Power of 22 microsatellite markers in fluorescent multiplexes for parentage testing in goats (Capra hircus). Animal Genetics. 30(6):431-437.

MACHUGH. D.E.; LOFTUS. R.T.; CUNNINGHAM. P.; BRADLEY. D.G. 1998 Genetic structure of seven European cattle breeds assessed using 20 microsatellite markers. Animal Genetics. 29:333-340.

MARSHALL, T.C.; SLATE, J.; KRUUK, L.; PEMBERTON, JM. 1998. Statistical confidence for likelihoodbased paternity inference in natural populations Molecular Ecology. 7(5):639-655.
MERIAUX. J.; ROGNON. R.; MAHLA. R.; OURAGH. L.; BOSCHER. M. 1998. Usefulness of microsatellite markers for parentage control and phylogenetic relationships in French and Moroccan horse breeds. XXVIth International Conference on Animal Genetics. Auckland, NZ. Section A. p.25.

MIROL, P.; PERAL-GARCÍA, J.L.; VEGA-PLA F.; DULOUT, N. 2002. Phylogenetic relationships of argentinean creole horses and other South American and Spanish breeds inferred from mitochondrial DNA sequences. Animal Genetics. 33:356-363.

MVSP (MULTIVARIATE STATISTICAL PACKAGE) 3.1. Disponible desde en Internet en: http://www.kovcomp.com/ (con acceso 7/4/06).

NEI, M. 1987 Molecular Evolutionary Genetics. Columbia University Press, NY.p.238-256.

RAYMOND M.; ROUSSET F. 1995. GENEPOP (version 1.2): population genetics software for exact tests and ecumenicism. J. Hered. 86:248-249.

RODAS, A. 2000. Variabilidad genética poblacional en equinos colombianos mediante microsatélites. Trabajo de grado Zootecnia. Universidad Nacional de Colombia sede Palmira. p.60-78.

RODERO, A.; DELGADO, J.V.; RODERO, E. 1992 Primitive andalusian livestock and their implications in the discovery of America. Arch. Zootec. 41(154):383-400.

ROMERO, M. 2000. Caracterización de la constitución genética de la población de caballos criollos colombiano mediante el estudio de frecuencias de marcadores microsatélites STRs. Trabajo de grado Biología. Universidad Industrial de Santander. p.35-50.

RON, M.; BLANC, Y.; BAND, M.; EZRA, E.; WELLER, J.I. 1996. Misidentification Rate in the Israeli Dairy Cattle Population and Its Implications for Genetic Improvement. J. Dairy Sci. 79(4):676-681.

RUIZ-GARCÍA, M.; TERWENGEL, PO.; PAYÁN, E.; CASTELLANOS, A. 2003. Genética molecular de 
poblaciones aplicada al estudio de dos grandes carnívoros neotropicales (Tremarctos ornattus Cuvier, 1825-Oso Andino y Panthera onca Linné, 1758-Jaguar): lecciones de conservación. Bol. Real Soc. Española de Historia Natural. Sección Biológica. 98:135-158.

SOLIS, A.; JUGO. BM.; MÉRIAUX. JC.; IRIONDO, M.; MAZÓN, J.I.; AGUIRRE, A.I.; VICARIO, A.; ESTOMBA, A. 2005. Genetic diversity within and among four south european native horse breeds based on microsatellite DNA analysis: Implications for conservation. J. Hered. 96(6):670-678.

STAHLBERGER-SAITBEKOVA, N.; SCHLÄPFER, J.; DOLF G.; GAILLARD, C. 2001 Genetic relationships in Swiss sheep breeds based on microsatellite analysis. J. Anim. Breeding Genetics. 118(6):379387.

TOZAKI, T.; TAKEZAKI, N.; HASEGAWA, T.; ISHIDA, N.; KUROSAWA, M.; TOMITA, M.; SAITOU, N.; MUKOYAMA, H. 2003. Microsatellite variation in Japanese and Asian horses and their phylogenetic relationship using a European horse outgroup. J. Hered. 94(5):374-80

TOZAKI, T.; KAHOI, H.; MASHIMA, S.; HIROTA, K.; HASEGAWA, T.; ISHIDA, N.; MIURA, N.; CHOIMIURA, N.; TOMITA, M. 2001 Population study and validation of paternity testing for thoroughbred horses by 15 microsatellite loci. J. Vet. Med. Sci. 63(11):1191-1197.
VIRGÜEZ, F. 2004. Aproximación a la caracterización morfométrica y fenotípica del caballo criollo de paso Colombiano. Tesis de grado. Universidad Nacional Facultad de Medicina Veterinaria y Zootecnia. Bogotá. p.41-45.

VIVAS, H. 1994. Evaluación de los problemas de aplomos en los caballos de paso fino de la Sabana de Bogotá. Tesis de grado. Universidad Nacional de Colombia. Facultad de Medicina Veterinaria y de Zootecnia. Bogotá. p.34-47.

WEBER, J.L.; MAY, PE. 1989. Abundant class of human DNA polymorphisms which can be typed using the polymerase chain reaction. Am. J. Hum. Genet. 44, 388-396.

WEIR, B.S.; COCKERHAM, C. 1984. Estimating F statistics for the analysis of population structure. Evolution 38:1358-1370.

WOMACK, JE. 2005. Advances in livestock genomics: Opening the barn door. Genome Research. 15(12):1699-1705.

ZABEK, T.; NOGAJ, A.; RADKO, A.; NOGAJ, J.; SLOTA, E. 2005. Genetic variation of Polish endangered Bilgoraj horses and two common horse breeds in microsatellite loci. J. Appl. Genet. 46(3):299330.

Recibido: Octubre 30 de 2006

Aceptado: Abril 23 de 2007 